\title{
DETERMINAÇÃO DE ALTITUGE ORTOMÉTRICA COM USO DA INTEGRAÇÃO DO GPS/NIVELAMENTO AO MAPGEO2010
}

José Milton Arana ${ }^{1}$, Daniel Arana ${ }^{2}$

${ }^{1}$ Faculdade de Ciências e Tecnologia - FCT. Departamento de Cartografia - UNESP - Presidente Prudente - SP. ${ }^{2}$ Curso de Graduação Engenharia Cartográfica - FCT - UNESP - Presidente Prudente - SP. E-mail: arana@fct.unesp.br

\section{RESUMO}

O Global Navigation Satellite System - GNSS, este sistema proporciona altitudes geométricas. No entanto, pelo fato das altitudes geométricas possuírem apenas significado matemático, normalmente não são utilizadas nas obras de engenharias, estas utilizam-se das altitudes ortométricas que estão vinculadas ao campo da gravidade da Terra. Com objetivo de verificar a aplicabilidade e confiabilidade executou-se levantamentos GPS em pontos de Referências de Nível - RRNN, pertencentes à Rede de Nivelamento Fundamental do Brasil - RNFB, determinadas pela Fundação Instituto Brasileiro de Geografia e Estatística - IBGE. Realizou-se levantamentos GPS em duas áreas distintas, uma situada na região de Maringá PR e outra na região de Presidente Prudente SP. Nas RRNN foram determinadas as ondulações geoidais, denominadas de GPS/nivelamento, nestas RRNN também foram determinadas as ondulações pelo MapGeo2010. A partir destas ondulações fez-se integração das ondulações GPS/nivelamento com as ondulações MapGeo2010. Com as ondulações determinadas por esta associação e as altitudes geométricas determinadas pelo GPS, calculou-se as altitudes ortométricas.

Palavras chaves: GNSS; altitude; altura geoidal; GPS/nivelamento.

\section{INTRODUÇÃO}

O Global NAVigation System with Time And Ranging/Global Positioning System NAVSTAR/GPS, este termo foi substituído por Global Navigation Satellite System - GNSS, mais abrangente, pois engloba os sistemas GPS, Global Orbiting Navigation Satellite System - GLONAS, Galileu entre outros (ALVES, et al., 2010). O GPS é um sistema de rádio-navegação, desenvolvido e realizado pelo United States Department of Defense da National Aeronautical Space Administration - NASA (SEEBER, 1993).

O método convencional, muito utilizado para a determinação da altitude ortométrica, é o nivelamento geométrico associado à gravimetria. Estes pontos são materializados no terreno e denominados de Referências de Nível (RN). Estas RRNN (plural de RN) são constituídas por peças de bronze chumbadas em blocos de concreto, na soleira de igrejas, monumentos, plataforma de estações ferroviárias, etc.

No entanto, o nivelamento com GPS, embora preciso, não fornece altitude ortométrica, e sim a altitude elipsoidal, que não possui significado físico, apenas matemático. As obras de 
engenharia necessitam de altitudes relacionadas ao geóide, limitando assim o uso do levantamento GPS.

Na grande maioria de obras de engenharia, nos levantamentos geodésicos ou topográficos, utilizam a altitude ortométrica (altitude referenciada ao geóide, este definido como sendo a superfície eqüipotencial do campo da gravidade que mais se aproxima do nível médio dos mares não perturbados). As altitudes, ortométrica e a geométrica, estão relacionadas pela equação (01).

$$
\mathrm{H} \cong \mathrm{h}-\mathrm{N}
$$

onde:

$\mathrm{H}$ - representa a altitude ortométrica, no ponto;

$\mathrm{h}$ - altitude geométrica; $\mathrm{e}$

$N$ - altura geoidal.

Este artigo tem como objetivo principal desenvolver apresentar uma metodologia de determinação da altitude ortométrica com uso do GPS, que poderá ser aplicada em qualquer região.

\section{FUNDAMENTAÇÃO TEÓRICA}

O GPS possibilita o posicionamento tridimensional e a determinação de posições horizontais precisas já é um processo rotineiro. Entretanto, a altitude geométrica, proporcionada pelo GPS, tem apenas um significado matemático e, geralmente, na cartografia e em obras de engenharia utiliza-se as altitudes ortométricas por possuírem um significado físico, diretamente relacionada com Trabalho.

\subsection{Precisão na Altitude}

A componente altitude é afetada pelo modo (técnica) de levantamento e pela observável utilizada no levantamento GPS. Utilizando-se de técnicas de levantamentos e de combinações de procedimentos de processamento, a precisão da altitude geométrica esperada é de 1,5 a 2 vezes menos acurada que as componentes horizontais (FEATHERSTONE, et al., 1998).

\subsection{Multicaminhamento}

Os multicaminhamentos ocorrem quando os sinais GPS são refletidos de objetos próximos, ou mesmo da superfície, antes de atingir a antena do receptor. Os multicaminhamentos podem causar erros de poucos metros na altitude elipsoidal quando utilizadas as observações do código, e 
de poucos centímetros quando utilizada a fase da portadora. Entretanto, o valor exato do erro provocado pelo multicaminhamento não pode ser determinado, pois este depende de fatores específicos do local. Para evitar os possíveis multicaminhamentos, adotou-se a estratégia de selecionar locais de rastreamentos em que não havia possibilidade das antenas receberem sinais refletidos de objetos (construções civis, arvores etc.) próximos.

\subsection{Orientação e Centro de Fase da Antena}

O centro de fase (eletrônico) da antena não coincide, necessariamente, com o seu centro geométrico. O centro de fase pode variar de acordo com a posição de cada satélite (elevação e azimute). As maiorias dos programas computacionais corrigem este efeito. Entretanto, com a finalidade de minimizar o efeito do centro de fase da antena em um levantamento, procedimentos especiais devem ser tomados, como utilizar preferencialmente antenas de um mesmo fabricante e mesmo modelo, e orientar todas as antenas na mesma direção nas coletas de dados GNSS.

\subsection{Processamento dos dados GPS}

Os dados pertencentes à região de Maringá foram processados com uso do "pacote" de programas denominado GPS Analysis Softhware - GAS, elaborado pela Universidade de Nottingham. Este programa foi desenvolvido, principalmente, para o processamento estático e ajustamento de multi-estações (STEWARD et al. 1994). O GAS foi elaborado visando principalmente o processamento de redes GPS, utilizando-se de dupla diferença de fase da portadora, onde a rede GPS é formada com estações que coletam simultaneamente os dados dos mesmos satélites (STEWARD et al. 1994).

No processamento dos dados GPS da Região de Presidente Prudente, foram utilizados os programas Reliance (da Ashtech) e GPSurvey (da Trimble), sendo que o primeiro (Reliance) foi utilizado para descarregar os dados do receptor ZXII e transforma-los para o formato RINEX; o segundo programa foi utilizado no processamento dos dados GPS, no modo estático relativo (observa-se que foram utilizados os dados, disponíveis, da estação UEPP, pertencente a RBMC).

\subsection{Determinação da ondulação geoidal a partir do GPS/nivelamento associado ao MapGeo2010}

A determinação da altura do geoidal a partir do rastreamento GPS em pontos pertencentes à rede fundamental de nivelamento do Brasil RN calcula a "real" ondulação do geoide. Os modelos do geopotencial fornecem a ondulação do geoide do modelo. A diferença entre as ondulações 
geoidal do modelo com as ondulação determinada com GPS/nivelamento, permite o cálculo da "separação" entre o modelo e o efetivo geoide ([] $N$ ); levado este conceito de diferenças de ondulações geoidais (modelo - GPS/nivelamento) às várias RRNN existentes na região, pode-se, com auxílio das Equações 02 e 03, do método dos mínimos quadrados (m.m.q.), determinar os parâmetros da Equação 05 (poli-superfície) que representa o "modelo matemático" da separação existente entre o geoide, naquela região, e o MapGeo2010.

Esquematicamente, a Figura 01 nos mostra a situação da determinação da ondulação do geoide por GPS/nivelamento associado ao MapGeo2010.

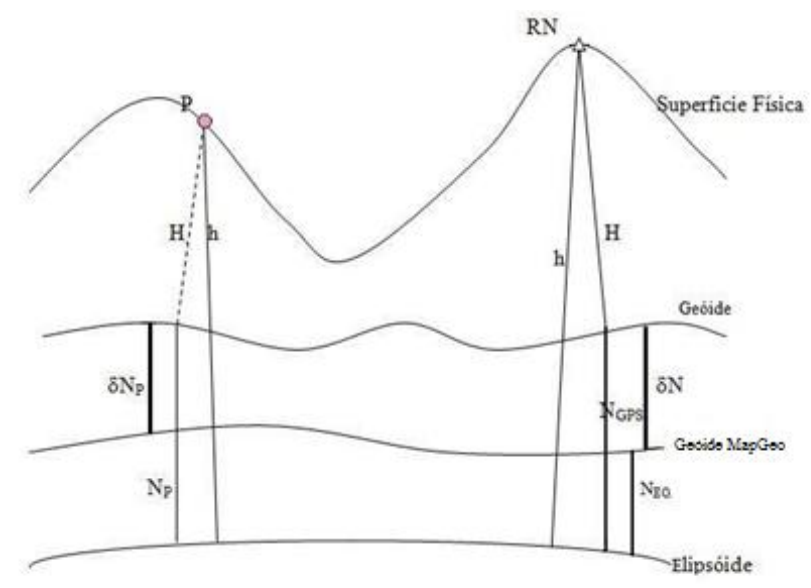

Figura 01. Determinação da ondulação do geoide pela associação do GPS/nivelamento ao MapGeo2010

Onde:

. H-Altitude ortométrica;

. h - Altitude geométrica;

. N NapGeo2010 - Ondulação do geoide obtida pelo modelo MapGeo2010;

. $N_{\text {GPS }}$ - Ondulação do geoide obtida pelo GPS/nivelamento; e

. 国N - Separação da ondulação determinadas por MapGeo2010 e por GPS/nivelamento.

Com auxílio da Figura 01 tem-se:

$$
\delta N=N_{G P S}-N_{M a p G e o 2010}
$$


Ainda, utilizando a Figura 01 e imaginando uma situação onde determinar a altitude ortométrica, utilizando desta técnica - associação do GPS/nivelamento com o modelo geopotencial - onde, $\mathrm{P}$ representa o ponto no qual intenciona-se a determinação do $\mathrm{N}_{\mathrm{p}}$. Em uma situação ideal, tem-se:

$$
N_{P}=N_{\text {MapGeo2010 }}+\delta N_{P}
$$

A determinação dos parâmetros da Equação $05(a, b, c, d)$ dá-se pelo método dos mínimos quadrados, onde serão determinados os parâmetros da Equação acima, que representará a diferença de ondulação ( $\left[N_{P}\right)$, obtido pelo MapGeo2010 e GPS/nivelamento nas RRNN.

$$
\begin{aligned}
& \delta N_{i}=N_{G P S}-N_{M a p G e o 2010} \\
& \delta N_{i}=a \varphi_{i}+b \lambda_{i}+c h_{i}+d
\end{aligned}
$$

Onde,

? $N_{i}$ - é a diferença de ondulação determinadas por GPS/nivelamento e da determinada pelo MapGeo2010, na $\mathrm{RN}_{\mathrm{i}}$;

$a, b, c, d$-parâmetros a serem determinados pelo

m.m.q.;

]$_{i}$ - latitude da $\mathrm{RN}_{\mathrm{i}}$;

]$_{i}$ - longitude da $\mathrm{RN}_{\mathrm{i}}$; e

$h_{i}$ - altitude geométrica da $\mathrm{RN}_{\mathrm{i}}$.

\section{DESENVOLVIMENTO}

Com a finalidade de atingir os objetivos deste projeto, foram selecionadas as regiões de Maringá PR e a de Presidente Prudente para as realizações dos experimentos de campo, onde as referências de nível (RRNN) pertencem à Rede de Nivelamento Fundamental do Brasil - RNFB (altitudes determinadas pelo IBGE). Foram aplicadas as recomendações sugeridas em (ARANA 2001). 


\subsection{Planejamento para o rastreamento na Região de Maringá PR}

Com as RRNN reconhecidas (encontradas) elaborou-se um programa de rastreio, o qual contém o itinerário de rastreio das RRNN. Na elaboração deste programa foram selecionadas as RRNN a serem rastreadas, procurando-se escolher as RRNN de maneira que ficassem aproximadamente eqüidistantes uma das outras. Nesta etapa foram selecionadas 48 RRNN.

\subsection{Planejamento para o rastreamento na Região de Presidente Prudente SP}

Considerando que as distâncias das RRNN mais afastadas da Estação UEPP, estação pertencente a Rede Brasileira de Monitoramento Contínuo - RBMC (considerada como estação conhecida no processamento GPS) estão à aproximadamente $50 \mathrm{~km}$, e sugestões de (ARANA 2001), verificou-se que 50 minutos de rastreamento nestas RRNN seriam suficientes para obter resultados confiáveis. Os tempos de rastreamentos das RRNN foram, aproximadamente, proporcionais às distâncias da estação UEPP.

\section{RESULTADO}

A tabela 01, que segue, contém: na primeira coluna a denominação da RN; na segunda coluna a discrepância entre as altitudes determinadas pelo nivelamento geométrico e as determinadas pela integração do GPS/nivelamento com o MapGeo2010. 
Tabela 01. Discrepâncias das altitudes IBGE e Integração Região de Maringá

\begin{tabular}{l|c}
\hline RN & $H_{\text {IBGE }}-H_{\text {Int }}$ \\
\hline $1582 S E$ & 0,0361 \\
\hline $1582 \mathrm{~N}$ & 0,1551 \\
\hline $1582 \mathrm{M}$ & 0,2323 \\
\hline $1582 \mathrm{H}$ & 0,2187 \\
\hline $1578 \mathrm{D}$ & $-0,0718$ \\
\hline $1578 \mathrm{GE}$ & $-0,1389$ \\
\hline $1578 \mathrm{JE}$ & $-0,0546$ \\
\hline $1578 \mathrm{P}$ & $-0,0001$ \\
\hline $1578 \mathrm{~S}$ & $-0,0070$ \\
\hline $1578 \mathrm{TE}$ & 0,0920 \\
\hline $1579 \mathrm{D}$ & $-0,0162$ \\
\hline $1579 \mathrm{~A}$ & $-0,0266$ \\
\hline $1778 \mathrm{UE}$ & $-0,1598$ \\
\hline $1594 \mathrm{~T}$ & $-0,0679$ \\
\hline $1594 \mathrm{~S}$ & $-0,1660$ \\
\hline $1594 \mathrm{R}$ & 0,0451 \\
\hline $1594 \mathrm{ME}$ & 0,0523 \\
\hline $1579 Z \mathrm{Z}$ & 0,0762 \\
\hline $1579 \mathrm{TE}$ & 0,0964 \\
\hline $1579 \mathrm{PE}$ & 0,1873 \\
\hline $1579 \mathrm{FE}$ & $-0,1732$ \\
\hline $1579 \mathrm{H}$ & 0,0133 \\
\hline $1591 \mathrm{JE}$ & $-0,0812$ \\
\hline $1591 \mathrm{HE}$ & 0,1140 \\
\hline & \\
\hline
\end{tabular}

\begin{tabular}{l|c}
\hline$R N$ & $H_{\text {IBGE }}-H_{\text {Int }}$ \\
\hline $1591 D$ & 0,0711 \\
\hline $1590 X$ & 0,0597 \\
\hline $1590 T E$ & 0,0516 \\
\hline $1590 R$ & $-0,0143$ \\
\hline $1590 \mathrm{LE}$ & 0,1063 \\
\hline $2926 \mathrm{U}$ & 0,0313 \\
\hline $2926 \mathrm{X}$ & 0,0530 \\
\hline $2927 \mathrm{CE}$ & 0,0915 \\
\hline $2927 \mathrm{E}$ & 0,1662 \\
\hline $2927 \mathrm{GE}$ & $-0,1198$ \\
\hline $2928 \mathrm{AE}$ & $-0,0055$ \\
\hline $2927 \mathrm{LE}$ & $-0,0680$ \\
\hline $2927 \mathrm{RE}$ & $-0,0720$ \\
\hline $2927 \mathrm{U}$ & $-0,1541$ \\
\hline $2928 \mathrm{~F}$ & $-0,0281$ \\
\hline $2928 \mathrm{H}$ & 0,1032 \\
\hline $2928 \mathrm{CE}$ & 0,1004 \\
\hline $2928 \mathrm{M}$ & $-0,2551$ \\
\hline $2928 \mathrm{PE}$ & 0,1102 \\
\hline $2928 \mathrm{SE}$ & $-0,0331$ \\
\hline $2928 \mathrm{UE}$ & $-0,0533$ \\
\hline $2928 \mathrm{VE}$ & 0,0805 \\
\hline $2928 \mathrm{ZE}$ & $-0,0536$ \\
\hline $2929 \mathrm{CE}$ & $-0,0490$ \\
\hline &
\end{tabular}

Na tabela 01, constata-se que a maior discrepância entre as altitudes da Região de Maringá, determinadas pelo IBGE e as determinadas pela integração do GPS/nivelamento ao MapGeo2010 é de 18,7cm, RN 1579PE (excêntrica) e a menor discrepância encontrada foi $-17,3 \mathrm{~cm}$, na RN 1579FE (excêntrica).

A tabela 02 contém, na primeira coluna as denominações das RRNN e na segunda coluna as discrepâncias entre as altitudes determinadas pelo IBGE e IGG e as determinadas pela integração GPS/nivelamento e MapGeo2010. 
Tabela 02. Discrepâncias das altitudes IBGE/IGG e Integração Região de Presidente Prudente

\begin{tabular}{l|c}
\hline$R N$ & $H_{\text {IBGE }}-H_{\text {Int }}$ \\
\hline $1525 \mathrm{t}$ & 0,4998 \\
\hline $1525 \mathrm{xe}$ & $-0,1160$ \\
\hline $1526^{\text {de }}$ & 0,2290 \\
\hline $1526 \mathrm{n}$ & 0,1067 \\
\hline $1526 \mathrm{xe}$ & 0,1690 \\
\hline $1584 \mathrm{~d}$ & 0,0565 \\
\hline $1584 \mathrm{~h}$ & 0,1059 \\
\hline $1584 \mathrm{r}$ & $-0,0640$ \\
\hline $1584 \mathrm{u}$ & 0,0339 \\
\hline $1585 \mathrm{ee}$ & 0,0439 \\
\hline $1525 \mathrm{~g}$ & 0,0242 \\
\hline $1585 \mathrm{he}$ & $-0,2087$ \\
\hline $32421 \mathrm{e}$ & $-0,2086$ \\
\hline igg3285e & 0,2844 \\
\hline
\end{tabular}

Analisando a tabela 02, verifica-se que a maior discrepância entre as altitudes determinadas pelo IBGE e as determinadas pela integração é na RN $1525 \mathrm{~T}$, valor de $50,0 \mathrm{~cm}$ e a menor discrepância determinada foi na RN 1585HE (excêntrica), com valor de 20,9cm.

\section{CONSIDERAÇÕES FINAIS}

As discrepâncias das altitudes determinadas pela integração do GPS/nivelamento ao MapGeo2010 e as determinadas pela Fundação Instituto Brasileiro de geografia e Estatística IBGE, na região de Maringá PR proporcionaram dispersão de 11,4cm. Na região de Presidente Prudente, a dispersão apresentada foi de $24,6 \mathrm{~cm}$. Acredita-se que esta notável diferença de dispersão seja devido às características geofísicas próprias da região e ao do número (quantidade) das RRNN. Na Região de Maringá foram utilizadas 48 e na Região de Presidente Prudente foram utilizadas 14 RRNN, ainda deve-se considerar a distribuição destas RRNN.

A utilização de um modelo matemático, para representar as discrepâncias das ondulações geoidais proporcionadas pelo GPS/nivelamento e as disponibilizadas pelo IBGE, determinado pelo método dos mínimos quadrados, deverá apresentar melhor resultados para as regiões que possuírem o maior número de observações. Assim, estas discrepâncias de maior amplitude nos extremos da região já eram esperadas.

Os resultados apresentados neste artigo estão compatíveis aos resultados apresentados em trabalhos de natureza similar a este (GREBNITCHARSKY, 2005) e que esta metodologia pode ser utilizada em várias aplicações da engenharia. 


\section{REFERÊNCIAS}

ARANA, J. M. O uso do GPS na elaboração de Carta Geoidal. Geociências. Editora Unesp. São Paulo. 2001. v 20. n 1/2.

BIRARD, G., SANTARSIERO, D., TUFILLARO, D., SURACE, L. Setting-up local "mapping geoids" with the aid of GPS/LEV Traverses Application to the geoids of Sardinia and Calabria. Journal of Geodesy. Springer-Verlag. Berlin.1995. v 70. n. 1-2.

GEMAEL, C. Introdução à Geodésia Física. Editora da Universidade Federal do Paraná Curitiba: Editora da UFPR, 1999.

GEMAEL, C e ANDRADE, J. B. Geodesia Celeste. Editora da Universidade Federal do Paraná. Curitiba. Curitiba. Editora da UFPR, 2004.

GREBNITCHARSKY, R.S.; RANGELOVA, E.V.; SIDERIS, M.G. Transformation between gravimetric and GPS/levelling-derived geoids using additional gravity information. Journal of Geodynamics. 2005. V 5.

KUANG, S., FIDIS, C., THOMAS, F. Modeling of the local geoid with GPS and leveling: A case study. Journal of Surveyind Engineering. Americn Society of Civil Engineers. 1999. v 125, n 3.

LEICK, A. GPS - Satellite Surveying. John Wiley \& Sons. New York. 1995. $2^{\text {a }}$ ed.

MONICO, J. F. G. Posicionamento pelo GNSS: descrição, fundamentos e aplicações. Editora Unesp. São Paulo SP. 2008.

SEEBER, G. Satellite Geodesy: Fundations, Methods and Applications. Walter de Gruyter, New York.1993.

ZONG, D. Robust estimation and optimal selection of polynomial parameters for the interpolation of GPS geoid heigts. Journal of Geodesy. Springer-Verlag. 1997. V 71. 